\title{
DISEÑO DE SITUACIONES DE APRENDIZAJE CENTRADAS EN EL APRENDIZAJE ESTRATÉGICO
}

\author{
Design of learning situations focused on strategic learning
}

\author{
Ronald José Feo Mora \\ e-mail: feoronald@gmail.com \\ Universidad Pedagógica Experimental Libertador. Venezuela
}

\begin{abstract}
RESUMEN
Las tendencias educativas en el siglo XX sobre los escenarios de enseñanza y de aprendizaje a pesar de sus avances, fueron dominadas y opacadas desde la praxis docente por un sistema de tradiciones de verificación de la conducta humana ante sistemas instruccionales que enaltecieron la hegemonía del profesor sobre la actividad mental de quién aprende, este accionar fortaleció una didáctica centrada en el ente de enseñanza, que arrinconó al estudiante a ser pasivo, a memorizar sin significados y sobre todo a aprender alejado de los motivos con lo cual el aprendizaje pertinente quedó en un serio cuestionamiento. De manera responsable ante el horizonte descrito, el propósito de este reporte de investigación es forjar escenarios de reflexión en los sujetos que fomentan la educación formal e informal de otro sujeto. La metodología empleada fue el análisis de contenido a partir del estado del arte del constructo situaciones de aprendizaje como criterio inicial de búsqueda, complementado con el enfoque del aprendizaje estratégico. Finalmente, se puede exponer que las situaciones de aprendizaje reconocen al estudiante como sujeto activo capaz de lograr autonomía para aprender y del profesor como mediador de procesos flexibles que favorecen saberes derivados de las competencias genéricas y específicas contempladas en el diseño curricular respectivo o ante la demanda del contexto donde el individuo se desempeña.
\end{abstract}

PALABRAS CLAVE: diseño; situaciones; aprendizaje; aprendizaje estratégico.

\begin{abstract}
The educational tendencies in the twentieth century on the teaching and learning scenarios, despite their advances, were dominated and opaque from the teaching praxis by a system of traditions of verification of the human behavior before instructional systems that exalted the hegemony of the teacher on The mental activity of those who learn, this action strengthened a didactic centered on the teaching entity, that cornered the student to be passive, to memorize without meanings and above all to learn away from the reasons with which pertinent learning was in a serious Questioning. In a responsible manner, the purpose of this research report is to forge reflection scenarios in the subjects that foster the formal and informal education of another subject. The methodology used was the analysis of content from the state of the art of the construct learning situations as an initial search criterion, complemented with the strategic learning approach. Finally, it can be stated that the learning situations recognize the student as an active subject capable of achieving autonomy to learn and of the teacher as mediator of flexible processes that favor knowledge derived from the generic and specific competences contemplated in the respective curricular design or the demand Of the context where the individual performs.
\end{abstract}

KEY WORDS: design; learning; situations; strategic learning.

Recibido/Received: 07/05/2017

Aprobado/Aproved: 18/12/2017

Cómo referenciar este artículo / How to reference this article:

Feo Mora, R. J. (2018). Diseño de situaciones de aprendizaje centradas en el aprendizaje estratégico. Tendencias Pedagógicas, 31, 187-206. doi: http://dx.doi.org/10.15366/tp2018.31.011 


\section{PREÁMBULO}

Los desafíos y las tendencias que giran alrededor de los ejes Tecnología de la Información y Comunicación (TIC), Economía y Política que impactan sobre los actores que conforman las sociedades del siglo XXI señalan que para impulsar un verdadero desarrollo humano se deben centrar esfuerzos en reconocer que los sujetos agrandan sus posibilidades para desarrollarse cuando tienen acceso libre y autónomo al conocimiento; que a su vez le permita desarrollar competencias influyentes para la mejora de sus vidas, esto exige en el sujeto la autoconsciencia de sus procesos de aprendizaje y sociales lo cual evoca al aprendizaje estratégico. Pero, el desarrollo humano visto desde el aprendizaje estratégico que fomenta en el sujeto la gestión del conocimiento, fragua una crisis pedagógica debatiendo rigurosamente la hegemonía de los esquemas y acciones educativas denominadas "tradicionalistas" que imponen una concepción del aprendizaje como un acto de estímulos y de respuestas o un hecho de comprobación de un contenido escolar, ignorando el mundo de los estudiantes, lo cual niega intereses comunes entre docentes y estudiantes.

Desde esta visión de la realidad educativa formal y no formal predominante descrita anteriormente, el autor de este reporte de investigación generó acciones para analizar el estado del arte del constructo situaciones de aprendizaje desde una mirada del enfoque del aprendizaje estratégico, y de este modo forjar un espacio de reflexión e intercambios de saberes en todo aquél sujeto que se encuentre inmerso de manera formal e informal en el proceso de planificación de la enseñanza. Es importante resaltar que el propósito enunciado anteriormente se mantuvo durante todo el quehacer investigativo, apoyado en una estrategia investigativa basada en el uso de la técnica de análisis de contenido y la experiencia docente del investigador, lo que permitió develar las características esenciales sobre el Diseño de Situaciones de Aprendizaje Centradas en el Aprendizaje Estratégico.

En consecuencia, se desarrolló una construcción teórica por parte del autor con la intencionalidad de generar acciones de vanguardia que permitan cimentar escenarios innovadores y de vigencia centrados en el ser humano que aprende, lo que no se opone a quién enseña, sino más bien, le otorga y recupera espacios de actividad y protagonismo acuñados desde nuestros ancestros al estudiante.

\section{DESARROLLO}

\subsection{Aprendizaje Estratégico: Construcción Autónoma del Aprendizaje}

Desde la tradición asociacionista y el funcionalismo se fundaron los conceptos de contigüidad, repetición, ensayo y error que colocaron al sistema nervioso en un lugar privilegiado antes de la cognición humana, como resultado de esta tendencia se excluyó que el conocimiento humano depende en gran medida de la actividad cognitiva, la cual es empleada para procesar la información del contexto y del almacenamiento significativo en la memoria; en consecuencia, se 
forjó la ignorancia que los motivos y el afecto son elementos fundamentales del aprendizaje humano.

El aprendizaje humano con la consolidación del enfoque cognitivo toma una redimensión y se fortalece, al reconocer que el conocimiento activado durante la solución de un problema, forma conceptos representados en estructuras lógicas conectadas entre sí, estas estructuras se encuentran almacenadas de manera significativa en la memoria a largo plazo, listas para ser transferidas al contexto que demande su activación. Con estas aserciones se reconoce al estudiante como un sujeto activo que construye el aprendizaje, ya que el aprendizaje no es aislado, se funda a través de la interacción con los pares, de la aprobación e influencia social, donde las acciones de cada sujeto están marcadas por una constante asimilación y contrastación entre los valores morales que impone el contexto y los pensamientos individuales del sujeto.

En correspondencia a las premisas anteriores Pérez y López (2000) afirman que en el aprendizaje intervienen procesos internos que promueven el procesamiento de nuevos conocimientos con los que ya se conocen, entre ellos se encuentran los cognitivos, los metacognitivos, los motivacionales y las conductas; estos permiten la construcción de estructuras mentales de manera efectiva y eficiente. El término efectiva significa que la persona posee la habilidad de alcanzar ciertos objetivos de aprendizaje. El término eficiente significa que la persona es capaz de lograr aprendizaje, mediante el uso óptimo de su tiempo, recursos y esfuerzo.

El aprendizaje humano visto desde la perspectiva de la construcción, de la auto estructuración, de la reconstrucción de saberes y de la organización interna de esquemas de manera efectiva y eficiente que se origina al entrar en conflicto lo que el sujeto ya sabe con lo que desea saber, admite que los sistemas educativos junto a sus actores clave forjen acciones concretas que promuevan la comprensión de la información de una manera significativa; de esta manera, la educación que se imparta será centrada en lo pertinente de la experiencia y no en la particularidad del contenido académico.

El término significativo se impone al aprendizaje de materiales sin sentido, tal como la memorización de pares asociados, de palabras o de sílabas, entre otras. Esta afirmación, puede ser entendida tanto como un contenido que tiene estructuración lógica inherente, como también aquel material que potencialmente puede ser aprendido de manera significativa (Batista y Oliveira, 1976). La posibilidad de que un contenido se torne con sentido depende de que sea incorporado al conjunto de conocimientos de un sujeto de manera sustantiva; o sea, afines a conocimientos preexistentes en la estructura cognitiva del sujeto. Este aprendizaje significativo es no arbitrario, en el sentido de que fue realizado con algún objetivo o teniendo en cuenta algún criterio. No arbitrario se opone a ipsis literis, o sea el tipo de aprendizaje que se manifiesta cuando el sujeto aprende los materiales sin darles sentido, sea porque no lo tienen, porque el sujeto no les confiere un sentido, por no tener nociones previas o una estructura mental adecuada a la cual incorporar los materiales o por no tener la intención de hacerlo. 
Entonces, se podría aseverar en palabras de Ausubel et. al. (1996) que el aprendizaje significativo ocurre cuando la nueva información se enlaza y relaciona con las ideas pertinentes de afianzamiento que ya existen en la estructura cognitiva del que aprende; por eso, con la inserción de esta concepción en la educación formal emergieron los criterios relacionados al sentido lógico y al sentido psicológico.

El sentido lógico es característico de los materiales mismos y solo con el tiempo y con un gran desarrollo psicológico el sujeto consigue captar completamente el sentido lógico de un material a aprender y darle una significación relacionándolo naturalmente en su cognición. En el sentido psicológico, las posibles relaciones entre conceptos que los sujetos pueden establecer casi indefinidamente, escapan al dominio del sentido lógico, es ahí donde la estructura psicológica del conocimiento con sentido tiene la capacidad de transformar el sentido lógico en sentido y comprensión psicológica, que es lo que el sujeto realmente hace en el proceso de aprendizaje, de esta manera el surgimiento del sentido psicológico depende no solo de que el sujeto posea como requisito experiencias previas, sino también depende de cada contenido en particular. Cuando el sujeto aprende proposiciones lógicamente significativas, no aprende el sentido lógico, sino el sentido que ellas tienen para él.

En consecuencia, a las aseveraciones que se vienen declarando, al ser comparadas con la realidad del Siglo XXI y sus demandas al contexto educativo, apuntaladas en las conferencias y los congresos mundiales como por ejemplo, la Declaración Mundial sobre Educación Superior (DMES, 1998), la Conferencia Mundial Sobre Educación Superior (CMES, 2009) y reafirmadas por el Informe Tendencias Sociales y Educativas en América Latina (ITSE, 2010) que declaran a la universidad como espacio público, la inclusión como fuente dignificante del ser, la actualización continua de competencias y sobre todo el avance tecnológico que implica una mayor conciencia del ser y su relación con el contexto, hacen recapacitar en profundidad sobre la promoción del aprendizaje significativo en los sistemas educativos actuales.

En este sentido, el aporte fundamental del aprendizaje significativo no es exclusivamente la consideración de las experiencias previas que posee el estudiante como tradicionalmente se ha pensado por parte de un gran segmento de los profesores, esta concepción que inicialmente plantea Ausubel et. al. (1996) resalta la pertinencia que deben poseer los medios didácticos, los cuales son en realidad según Leontiev (1978) instrumentos didácticos materiales, generadores de un proceso de comunicación didáctica mediada y coherente que en conjunción a las experiencias previas del estudiante y al significado personal que este le incorpora a la información que procesa, erige un sentido psicológico pertinente para que el sujeto aprenda. Así pues, el estudiante es un ser humano capaz de construir su aprendizaje a través del sentido psicológico que es activado por el sentido lógico que poseen los instrumentos didácticos materiales. A manera ilustrativa se presenta la figura siguiente: 


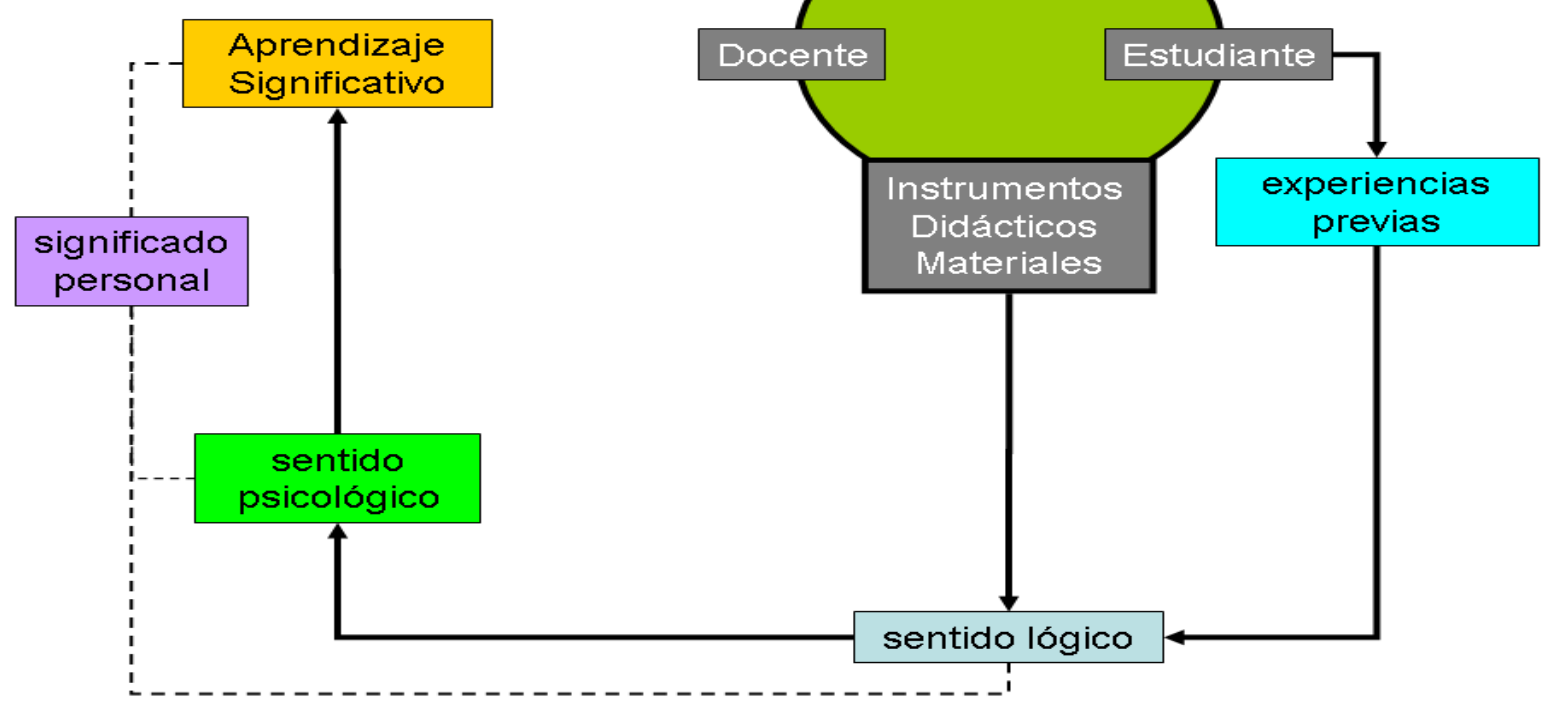

Gráfico 1. El Aprendizaje Significativo

En el hecho educativo se evidencia una realidad que desdibuja las buenas aspiraciones del colectivo profesoral y estudiantil, se da énfasis en promover una verdad preexistente donde los estudiantes deben escuchar, comprender y proceder, desvalorando la construcción colectiva de los saberes y el significado personal que cada estudiante le incorpora a lo que aprende, auspiciando de esta manera un abandono del fomento del sentido psicológico; es decir, un auténtico aprendizaje significativo. Al mismo tiempo, el profesor declara y ejerce acciones desde su experiencia donde se presume que dichas acciones promoverán la construcción de aprendizajes; sin embargo, los saberes emergentes producto de la reflexión, de la creatividad y de los saberes previos de los estudiantes, pocas veces es valorado para promover la construcción del conocimiento que hará del sujeto un ente activo socialmente, capaz de darle respuestas a un contexto que se lo exige.

Estas ideas descritas permiten alegar que dentro de las instituciones educativas se está realizando algo de manera distinta a la realidad del sujeto. La didáctica soporte esencial de la enseñanza promueve la activación de procesos cognitivos, de habilidades y de valores diferentes a los requerimientos del contexto, lo que ocasiona un déficit estratégico en el estudiante y la no valoración del aprender permanentemente.

En otras palabras, los escenarios educativos y sus actores clave están llamados a realizar un alto en su praxis diaria y centrarse a observar las actividades, las operaciones individuales y las colectivas que emplean los 
profesores y los estudiantes durante los procesos de enseñanza y aprendizaje. Esto supone que dentro de las acciones propias del sistema educativo formal y no formal se debería incorporar la metacognición y las estrategias de aprendizaje; es decir, promover el aprendizaje estratégico que conlleve a una valoración de la autonomía al aprender. Esto último con el anhelo de generar una formación para la vida misma, centrada en la promoción de procedimientos más que de contenidos académicos. Aprender de manera consciente sobre la base de estrategias es un beneficio indeleble.

Estas afirmaciones indican que un estudiante estratégico que planifique, revise y autorregule sus operaciones mentales y de estudio antes, durante y después de afrontar una situación de aprendizaje, junto a sus experiencias previas, podrá construir el sentido psicológico a la información que procesa, lo que definiría su aprendizaje en significativo. Esta premisa refleja lo actual de la promoción del aprendizaje estratégico, lo que consentirá la toma de consciencia y la valoración de la autorregulación del aprendizaje como elemento fundamental del currículo escolar y de las formas de aprender consideradas no formales e informales (los grupos y redes primarios, la comunidad local y el ciberespacio) que permitan aprender de manera permanente. Finalmente, el aprendizaje estratégico fortalece la didáctica escolar y como ya se mencionó las acciones no formales e informales de aprender al proponer amplios elementos para conformar acciones promotoras de los procesos cognitivos y de estudios, centradas en el uso de la metacognición y las estrategias de aprendizaje, lo que origina como secuela la formación de sujetos autónomos preciados en este siglo XXI.

\subsection{Diseño de Situaciones de Aprendizaje}

El diseño de situaciones de aprendizaje concreta las acciones básicas y los elementos que constituyen una estrategia con fines educativos que involucran e impulsan acciones comunicativas mediadas y flexibles entre los agentes de enseñanza y los agentes de aprendizaje bajo una disposición orientada hacia el alcance de metas comunes que favorezcan el proceso de aprendizaje. De ahí que las acciones básicas del profesor que asume las premisas antes expuestas, se direccionan hacia el fomento de procedimientos para que el estudiante desde la activación de sus procesos cognitivos de manera autorregulada construya y genere la autoconsciencia sobre sus habilidades y destrezas ante una tarea escolar. Las proposiciones expuestas permiten el reconocimiento del estudiante como un sujeto que posee una actividad mental capaz de generar acciones autónomas para aprender de manera pertinente ante los desafíos y retos propios del contexto social donde este hace vida.

En este sentido Mendia (1989) sugiere que es evidente un cambio de posicionamiento del profesor con respecto al diseño de acciones educativas centradas en el enseñante, hacia un accionar centrado en situaciones que faciliten la activación de las capacidades cognoscitivas, propicie las estrategias para solucionar problemas y desarrolle las capacidades socio-afectivas, puesto que 
éstas representan el elemento central para lograr competencias personales y profesionales.

De acuerdo con Herrera (1991) hablar de "situación" en el ámbito educativo se refiriere a la concepción del marco ecológico, contextual y relacional en la interacción e interrelación de las personas que intervienen en los procesos de enseñanza y de aprendizaje. El marco ecológico recoge el hábitat escolar natural físico que acoge el espacio - temporal y humano donde emergen las interacciones entre los actores de los procesos de enseñanza y aprendizaje. El marco contextual, recoge una serie de peculiaridades específicas que lo hacen único e irrepetible y el marco relacional, recoge las interacciones e interrelaciones humanas que se dan en él y que condicionan explícita e implícitamente todo el proceso educativo y su posterior aplicación en la vida real.

Los sucesos emergentes de la acción educativa enmarcados en una situación ecológico-contextual-relacional quedan restringidas exclusivamente a dos: (a) la socializada; y (b) la individualizada. No se puede olvidar que, aunque la individualidad representa la identidad particular del hombre y es la base de su personalidad, se hace persona a través del contacto con los demás conformando su identidad grupal (Herrera, ob.cit.).

La situación ecológica - contextual - relacional es esencial su consideración en el diseño de situaciones de aprendizaje que fomenten autonomía escolar, ya que si un profesor no posee claridad de los marcos característicos donde emerge la acción de aprender, podría no orientar su accionar de enseñanza de manera adecuada y por ende ser obstáculo en la mediación del conocimiento y del proceso de comunicación.

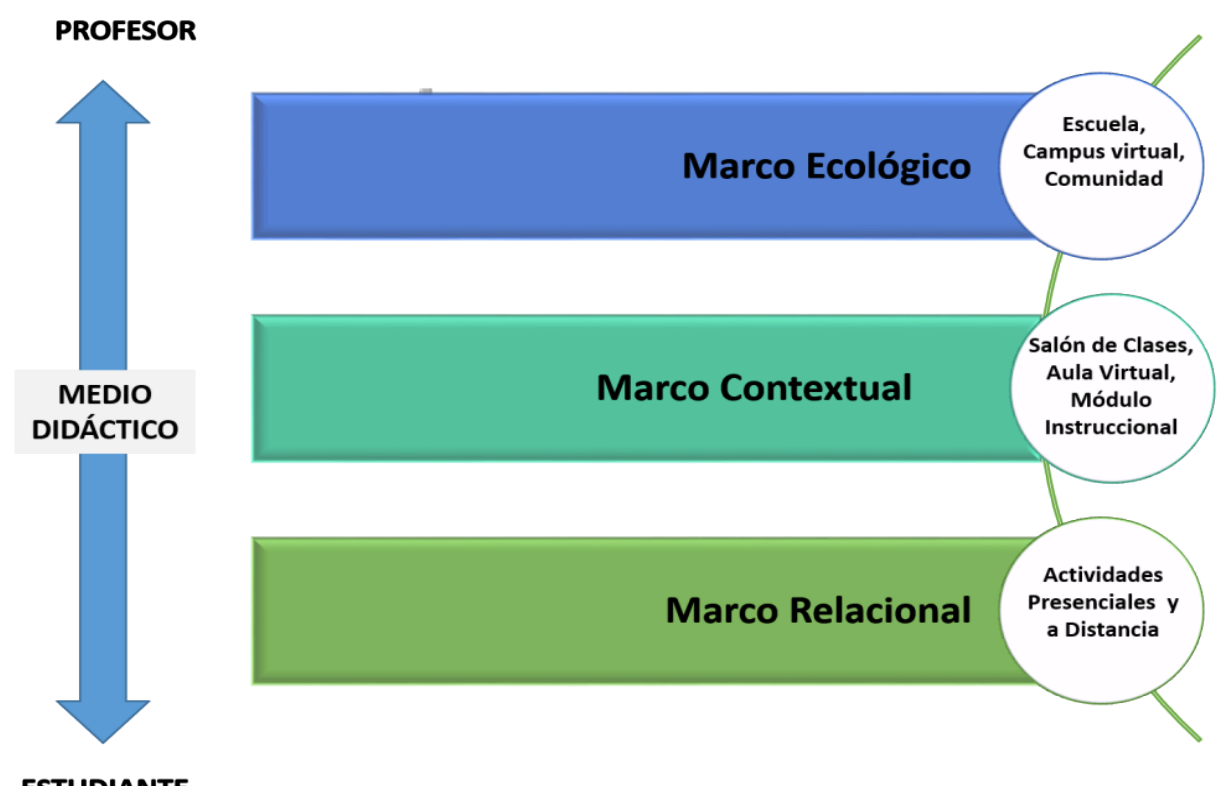

Figura 2. Situación Ecológico-Contextual-Relacional. 
Partiendo de los supuestos anteriores, Marzano (1999), Chan y Tiburcio (2000) admiten que la información es un insumo del conocimiento, pero es la actividad sobre la información lo que produce el aprendizaje. De ahí que contenidos y actividad se deban integrar al diseñar una situación con fines formativos. Se puedan combinar de la manera siguiente: (a) contenidos en función de las actividades de aprendizaje; y (b) actividades de aprendizaje en función de los contenidos. Estas combinaciones deben considerar, según los autores mencionados, cinco (5) dimensiones, las cuales pueden ser descritas como:

1.- Problematización-Disposición, implica que el estudiante reconozca las necesidades formativas que tiene. Equivale a una fase de interpelación sin la cual es difícil que el sujeto pueda iniciar un proceso de aprendizaje, pues el carácter de cuestionamientos, curiosidad inicial o la motivación no se genera en él y su aplicación al estudio puede obedecer a finalidades no necesariamente ligadas al conocimiento.

2.- Adquisición - Organización del Conocimiento, contempla las conexiones que los estudiantes hacen de la información, aquello nuevo que requiere un punto de enlace con lo ya sabido para significar algo. Así mismo esta integración informativa se hace con base en una organización, de modo que toda información es acomodada de acuerdo a determinados esquemas.

3.- Procesamiento de la Información, supone que el aprender implica operar sobre la información; es decir, desarrollar operaciones mentales tales como: (a) el análisis; (b) la inducción; (c) la comparación; (d) la clasificación; (e) la síntesis y entre otros procesos cognitivos. Operaciones que constituyen una base de pensamiento que habilita al sujeto para trabajar con todo tipo de información.

4.- Aplicación de la Información, el ciclo del aprendizaje se consolida en la medida que la información se pone en juego para tratar con problemas reales o posibles. Hacer prácticas, operar los conceptos, investigar, planear el proyecto, resolver el problema y estudiar casos, entre otros ejercicios, permite utilizar los conocimientos adquiridos de manera significativa.

5.- Consciencia del Proceso de Aprendizaje- Auto Evaluación, el sujeto que hace conciencia de la forma como aprende, de los pasos que sigue, que controla cada dimensión y se da cuenta del trayecto de la información, las operaciones y usos de la misma, consigue una estrategia para aprender y con ello su formación puede darse autogestivamente. 


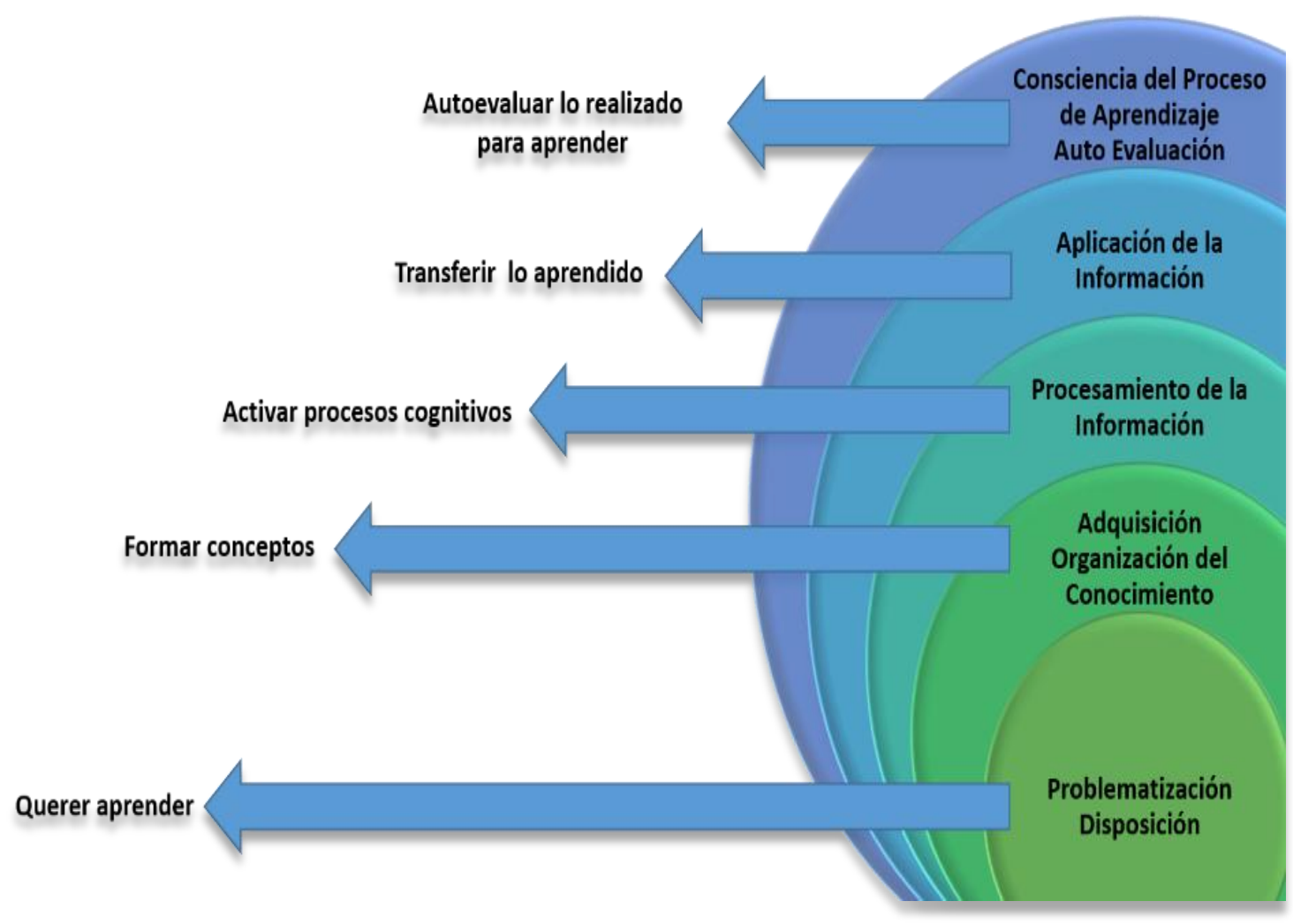

Figura 3. Dimensiones del Aprendizaje.

Una situación es un hecho o acontecimiento social o natural que ocurre en el entorno del estudiante, se convierte en una situación de aprendizaje cuando se usa con fines formativos; es decir, se proyecta en la acción educativa que ejerce el profesor para propiciar el aprendizaje mediante operaciones ordenadas y articuladas en secuencias. La situación ocurrida en el entorno puede simularse, reconstruirse, escenificarse o bien analizarse a través de un video, película, nota informativa, registro fotográfico o visita al lugar de los hechos. Por lo tanto, las situaciones de aprendizaje que ocurren durante la acción educativa son escenarios que pueden aprovecharse para generar conocimientos, desarrollar competencias, habilidades, destrezas, actitudes y valores (Flores, 2009).

Posiblemente en la presente década, la consolidación de los currículos por competencias ha generado la demanda y la aceptación de un profesor que ejerza acciones mediadoras y facilitadoras de los aprendizajes lo que ha originado un conjunto de implicaciones para la didáctica y para la planificación de dichos aprendizajes. El profesor proyecta condiciones que aseguren un camino flexible para la construcción de significados, en un tiempo y espacio, donde prever esos escenarios recae en buena parte sobre él, siendo este quien tiene a su cargo la administración de la unidad curricular a través de espacios presenciales o a distancia. El escenario descrito apunta hacia el fomento de la autonomía escolar, 
lo que es igual a la inserción del aprendizaje estratégico como enfoque para el diseño de situaciones de aprendizaje.

Las situaciones de aprendizaje pueden ser definidas como actividades educativas diseñadas por el profesor con el objetivo de potenciar en el estudiante la construcción autónoma y responsable de competencias genéricas y específicas, reguladores de una actuación profesional eficiente y ética en un ambiente participativo y dialógico (Gonzales et al., 2011).

Para Piraval, Morales y Gutiérrez (2013) las situaciones de aprendizaje son momentos, espacios y ambientes organizados por el profesor, en los que se ejecuta una serie de acciones educativas, que estimulan la construcción de aprendizajes significativos y propician el desarrollo de competencias en los estudiantes, mediante la resolución de problemas simulados o reales de la vida cotidiana. Estas acciones educativas suelen ser caracterizadas por: (a) entornos complejos que impliquen un desafío para el aprendizaje y tareas integradoras; (b) negociación social y responsabilidad compartida como parte del aprendizaje; (c) representaciones múltiples del contenido; (d) comprensión de que el conocimiento se elabora; y (e) enseñanza centrada en el estudiante.

Herrera (1991), Gonzales et al. (2011) y Piraval et al. (2013) coinciden en describir las cualidades del profesor desde la concepción del diseño de situaciones de aprendizaje, como un agente facilitador, mediador, guía, líder, organizador, investigador, evaluador y tutor. Donde dichas cualidades le permiten tomar consciencia sobre los procesos que activan los estudiantes al aprender a la par del desarrollo de competencias para el diálogo mediador y promotor de la solución de problemas, el razonamiento y la aplicación de conocimientos que motiven a los estudiantes al trabajo individual y grupal.

Igualmente, los mismos autores coinciden en afirmar que las situaciones de aprendizaje se centran en el estudiante, donde es protagonista, participativo, propositivo, creativo, activo, curioso, investigador y colaborativo al participar activamente en el desarrollo de situaciones simuladas o reales para las que propone soluciones o alternativas. Acepta desafíos, sabe trabajar en equipos colaborativos y es un participante espontáneo y diligente en la construcción individual y grupal del conocimiento.

Para Piraval et al. (2013) las situaciones de aprendizaje se diseñan a partir de la planificación que el profesor realiza, en consideración de las orientaciones curriculares propias del nivel educativo en donde se desarrollaran. La planificación incluye las competencias de área, de ejes y de grado o etapa que interesa desarrollar en los estudiantes; los indicadores de logro y los conocimientos declarativos, procedimentales y actitudinales que servirán como medio para lograr los resultados propuestos. Las situaciones responden a la planificación de los aprendizajes para facilitar a los estudiantes, experiencias de aprendizaje significativas. En este sentido los mismos autores señalan algunos aspectos técnicos - pedagógicos a considerar al diseñar situaciones de aprendizaje: (a) contexto, representado por las características y necesidades educativas, culturales, lingüísticas y sociales de los estudiantes; (b) ambiente de aprendizaje, 
representado por el clima afectivo que fortalece la identidad, la autoestima y la convivencia armónica entre los estudiantes y los profesores; y (c) organización de los estudiantes, representado por las formas de interacción de los estudiantes al aprender que propician un intercambio académico, social y cultural. Como ilustración, se muestra la siguiente gráfica:

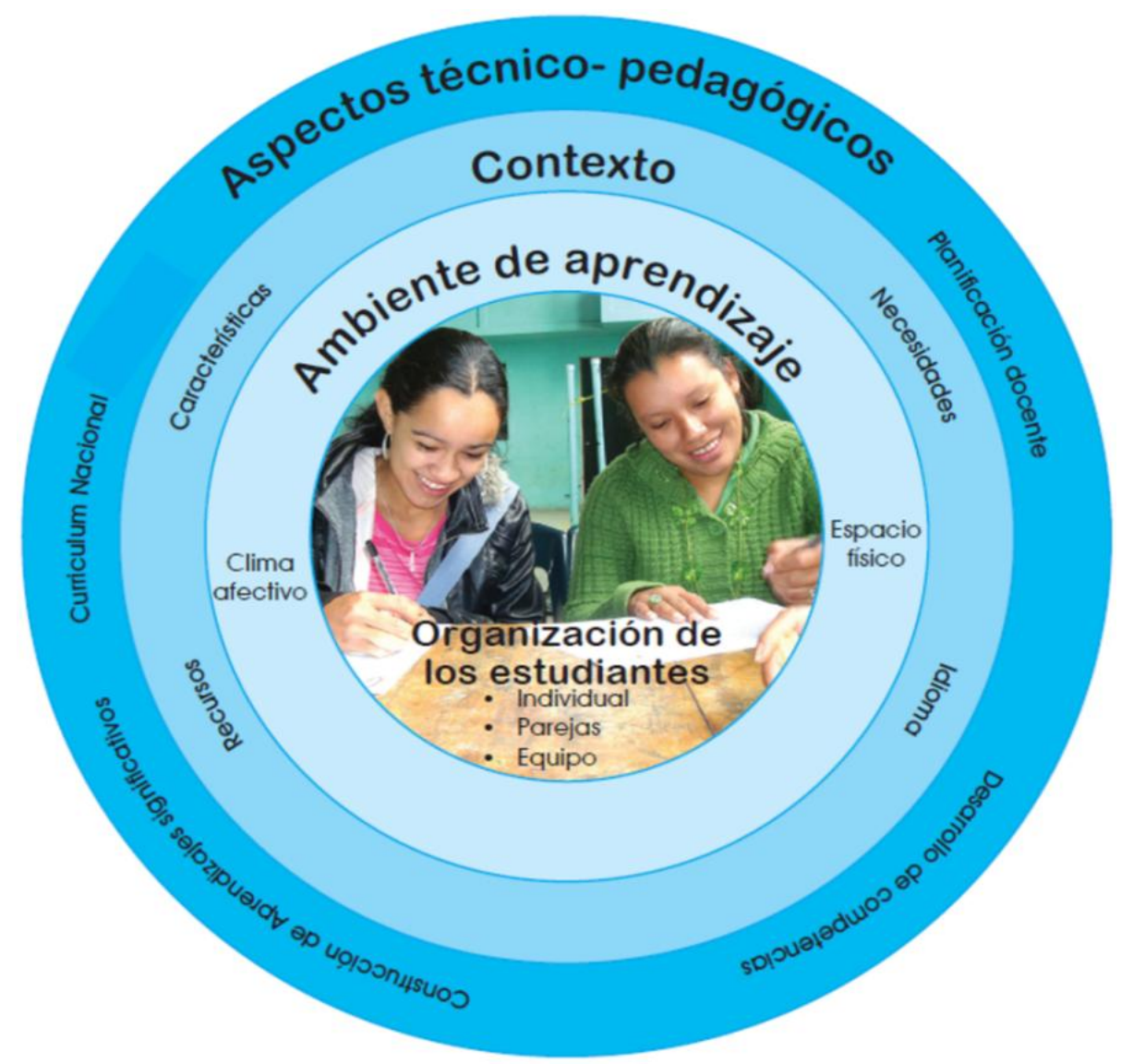

Figura 4. Aspectos Técnicos - Pedagógicos a Considerar al Diseñar Situaciones de Aprendizaje. Tomado de: Piraval, M., Morales. B., y Gutiérrez. M. (2013). Situaciones de Aprendizaje, Pautas Metodológicas Para el Desarrollo de Competencias en el Aula. Guatemala: dirección General de Currículo.

Es importante mencionar que Feo y Guerra (2014) y Feo (2014) concuerdan en proponer una serie de aserciones teóricas para ser consideradas en la administración de unidades curriculares o en el diseño de situaciones de aprendizaje ante contextos educativos presenciales y a distancia, los cuales se presentan a continuación:

1.- El conocimiento es una construcción subjetiva desde la realidad cultural, moral y lingüística de cada individuo que la conforma.

2.- La interpretación del contexto genera la posibilidad de que todo sujeto se desarrolle de manera personal y social. 
3.- El conocimiento humano se manifiesta como una interacción sujetoobjeto, que transita de la ignorancia al saber en una actividad mental infinita intrínsecamente mediada por la praxis social.

4.- Los sistemas sociales no son entidades que estén ubicados en un lugar en el espacio y en el tiempo, se trata de una persistente conciliación entre sujetos, que los hacen reales y que origina efectos reales.

5.- Todo sujeto debe ser estimado como un ser capaz de desarrollar la conciencia sobre su propio pensamiento para formar conceptos significativos.

6.- El profesor se encuentra orientado a generar situaciones de aprendizaje para que el estudiante despliegue una actividad mental constructiva rica en significados y diversa en ideas.

7.- La enseñanza es un proceso socializador que permite al sujeto apropiarse del contexto donde ha nacido a través de la mediación de situaciones de aprendizaje por parte del profesor.

8.- El aprendizaje es un proceso psicológico, activo, constructivo e individual, lo que genera una estructura mental única en cada sujeto compuesto por experiencias, conceptos, sentimientos y creencias sociales.

En consonancia de lo descrito y explicado sobre el diseño de situaciones de aprendizaje centradas en el enfoque del aprendizaje estratégico, es primordial resaltar que ceder el control de las acciones educativas de manera progresiva en el estudiante, significa un compromiso ético y moral centrado en lo humano; además, de un esfuerzo cognitivo por planificar estrategias desde y para el estudiante que le motiven a aprender con significado. Desde esta visión integradora, sistémica y dialógica de una didáctica centrada en el que aprende y no en el que enseña emerge el diseño de situaciones de aprendizaje para la construcción autónoma del aprendizaje. Lo cual contraria y potencia hacia la vanguardia de los viejos esquemas y modelos para enseñar y aprender que aún a casi dos décadas del siglo XXI predominan la praxis educativa formal y no formal.

\subsection{Diseño de Situaciones de Aprendizaje Centradas en el Aprendizaje Estratégico}

El diseño de situaciones de aprendizaje se define como momentos, espacios, actividades y ambientes organizados por el profesor con el objeto de promover en el estudiante la construcción autónoma y responsable de saberes, para consolidar las competencias genéricas y específicas que regulan una actuación profesional eficiente y ética en un ambiente participativo y dialógico que es desarrollado en un marco ecológico, contextual y relacional concebidos estos como el centro educativo, el encuentro pedagógico y las tareas integradoras. El aprendizaje estratégico es una acción deliberada y reflexiva por parte del estudiante de sus estrategias cognitivas, metacognitivas y sociales que le facilitan 
el procesamiento de la información y posterior almacenaje en la memoria significativa, este proceso ocurre cuando el estudiante se enfrenta ante las demandas intelectuales que implican la solución de una tarea integradora implícita en una situación de aprendizaje.

Asumir que los procesos educativos deben girar alrededor del proceso de aprendizaje forja el escenario para que el estudiante sea el epicentro de la formación, sin menoscabo de la labor de enseñanza del profesor que ejerce acciones de mediador y tutor. Desde la visión que el estudiante y su aprendizaje es epicentro del proceso educativo de manera contextualizada, una situación de aprendizaje centrada en el aprendizaje estratégico, tiene las siguientes características:

1.- Posee esencialmente cuatro (4) elementos técnicos - pedagógicos definidos por el contexto, el ambiente de aprendizaje, el procesamiento de la información y las tareas integradoras ordenados de una manera coherente hacia las expectativas del estudiante para conforman un dialogo didáctico mediado que establece un puente flexible de comunicación entre profesor y estudiante al igual que con los estudiantes y sus propios compañeros.

2.- Los elementos técnicos - pedagógicos no se pueden asumir como una secuencia de fases que responden a un modelo de diseño instruccional, más bien son planos de concreción de las ideas y análisis iniciales realizados por el profesor para que el estudiante desde sus motivos que le impulsan a aprender pueda configurar sus estrategias de aprendizaje.

3.- Las situaciones de aprendizaje generan acciones cognitivas, afectivas y emocionales en los estudiantes pertinentes para aprender en sintonía con la autoconsciencia, la cual es activada y mediada por los propios elementos que la constituyen.

4.- Las situaciones de aprendizaje no son tareas rígidas donde se requiere una conducta observable, sino un accionar que enlaza elementos y pensamientos para generar una invitación a construir y conocer la realidad desde la perspectiva individual y colectiva que favorezca el desarrollo de competencias. 


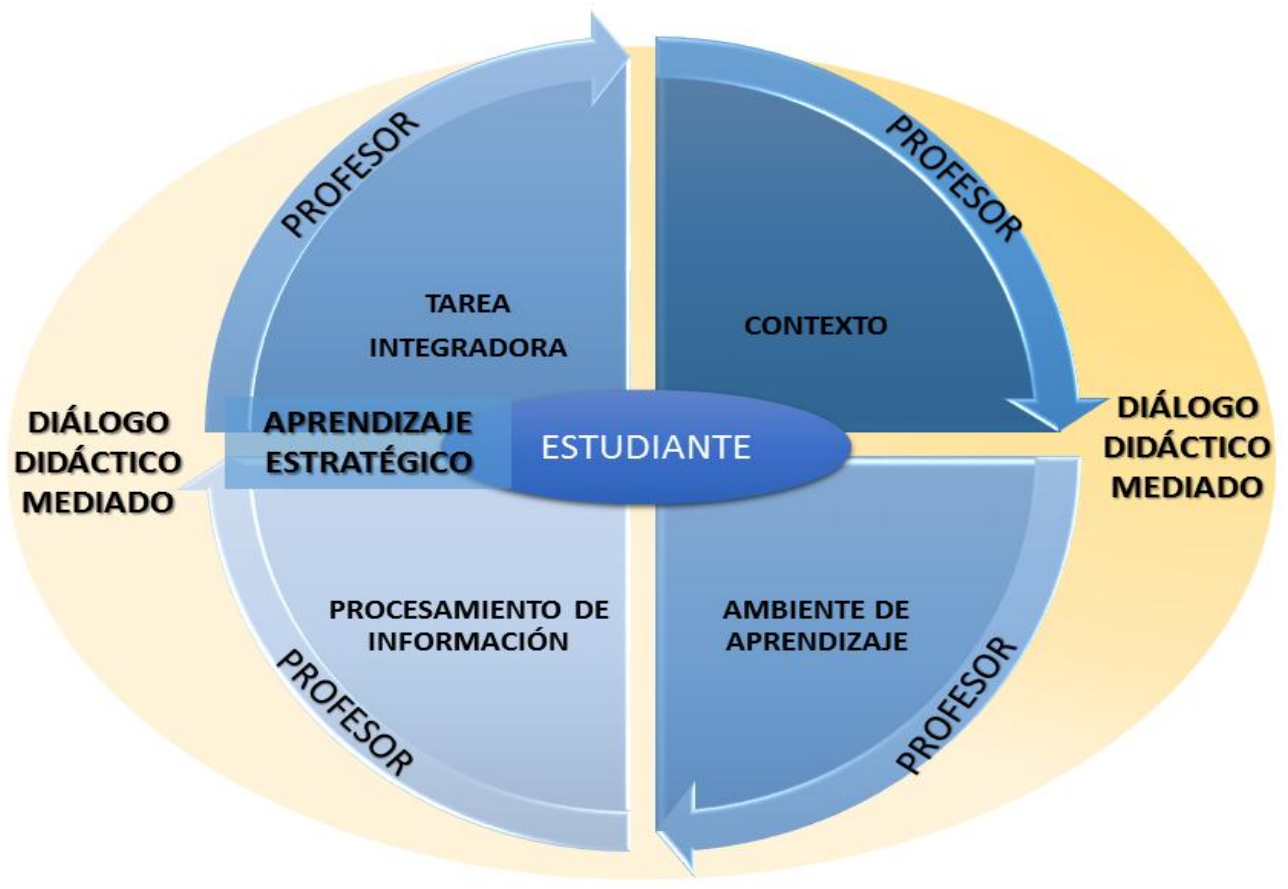

Figura 5. Diseño de Situaciones de Aprendizaje

El Dialogo Didáctico Mediado representa al proceso de comunicación didáctica que materializa la interacción entre estudiante y profesor permitiendo hacer realidad el encuentro pedagógico. Sin la presencia de un diálogo didáctico mediado no hay interacción pertinente y significativa fomentadora de la autonomía escolar.

El contexto queda definido por la premisa que declara que todo estudiante posee la capacidad de autorregular su aprendizaje, desarrollar aprendizaje autónomo y colaborativo que lo presenta e impone como un sujeto activo. Pero para determinar los niveles y rasgos de autonomía del estudiante el profesor debe abrir espacios que permitan analizar las características y necesidades que posee el estudiante a través de las variables: (a) necesidades de los estudiantes; (b) clima afectivo; (c) entorno socioeconómico; (d) recursos materiales y tecnológicos; (e) nivel educativo; y (f) duración/tiempo.

El profesor puede considerar las siguientes interrogantes como guías para un mayor acercamiento a la realidad vivida por el estudiante: ¿Cómo es el escenario en el que se va a desarrollar las situaciones de aprendizaje?, ¿Qué duración tendrá el desarrollo de cada situación de aprendizaje?, ¿Qué conocimientos previos poseen los estudiantes?, ¿Cuál es el estilo de aprendizaje de los estudiantes?, ¿Cuáles serán las expectativas de los estudiantes ante las situaciones de aprendizaje?, ¿Cómo serán las estrategias de aprendizaje de los estudiantes?, ¿Cuál será el nivel educativo a implementar el diseño de situaciones de aprendizaje? y ¿Cuántos estudiantes son?

El ambiente de aprendizaje es el espacio en donde se concretan las acciones de enseñar y aprender; es decir, se llevan a cabo las relaciones entre 
profesor y estudiante o entre estudiante y estudiante. El ambiente de aprendizaje debe generar un espacio receptivo, familiar, de respeto, de comunicación que motive al estudiante hacia la autonomía escolar que lo ayuden al logro de los propósitos de la unidad curricular y el alcance de las metas propias del estudiante. Es necesario indicar que el ambiente de aprendizaje puede ser combinado por marcos contextuales presenciales y a distancia o virtuales. Además, un ambiente de aprendizaje podría ser denominado como una situación de aprendizaje macro constituida por diversas situaciones de aprendizajes a nivel micro que alojan o proponen tareas integradoras. Finalmente, el ambiente de aprendizaje posee las orientaciones necesarias y los medios didácticos pertinentes para el desarrollo de una situación de aprendizaje.

El profesor para diseñar un ambiente de aprendizaje que responda al análisis contextual debe abordar la mayoría de las siguientes interrogantes: $¿ \mathrm{EI}$ profesor tiene interés en los procesos de tutoría?, ¿Cómo se fomentará el aprendizaje estratégico?, ¿Cuáles contenidos pueden ser abordados con mayor facilidad para promover aprendizaje estratégico?, ¿Cuáles medios didácticos se seleccionaran?, ¿Cómo se fomentará el diálogo didáctico mediado?, ¿La gestión de la unidad curricular facilita el fomento de la comunicación didáctica y el aprendizaje estratégico?, ¿Cuáles acciones se deben valorar de manera formativa y cuáles de manera final?, ¿Cómo se harán las prácticas para desarrollar habilidades y concretar conocimientos? y ¿Cuál será la competencia genérica a desarrollar?.

El procesamiento de la información se encuentra representado por las implicaciones que se prevén deben emerger durante el procesamiento de la información; en otras palabras, el procesamiento de la información implica activar procesos cognitivos como actividad mental donde la mediación del profesor, conjuntamente con la información que expone el medio didáctico fomentan en el estudiante el reconocimiento consciente de los procesos cognitivos que le facilitan procesar la información y comprenderla con significados. Las instrucciones, demandas y las actividades inmersas en la situación de aprendizaje guían al estudiante hacia cuáles procesos cognitivos activar y hacia los procedimientos de estudio a ejecutar como apoyo a sus estrategias de aprendizaje.

Las interrogantes sugeridas para este plano son: ¿Cómo se promoverá la activación de procesos cognitivos en el ambiente de aprendizaje?, ¿Cómo se captará la atención del estudiante en cada situación de aprendizaje?, ¿A partir de dónde se promoverá la metacognición?, ¿Cuáles son los procesos cognitivos que deben activar los estudiantes para comprender los contenidos e instrucciones? y ¿Será factible que el estudiante emplee otros procesos cognitivos no contemplados?

Las tareas integradoras son labores insertas en un marco relacional donde los estudiantes concretan los conocimientos, las habilidades, los hábitos, los valores y la ética que propician una competencia específica ante una situación concreta que se aproxima a un problema ajustado al contexto profesional o social en donde se desenvuelve el estudiante. A través de las tareas integradoras se lleva a cabo el proceso de evaluación de los aprendizajes, por lo que de manera 
operativa, coherente y pertinente las tareas integradoras se insertan en la estrategia de evaluación. Otra característica esencial de las tareas integradoras es que responden a un contenido específico que paulatinamente llevan a escenarios de mayor complejidad en función a las competencias que se pretenden construir en la unidad curricular.

Cómo guía al diseño de las tareas integradoras de manera pertinente y en favor al estudiante se recomiendan las siguientes interrogantes: ¿Se logra la concreción de las habilidades, los conocimientos, los valores, las competencias y la ética en la tarea integradora?, ¿Cuánto tiempo y recursos se debe emplear para su solución?, ¿La tarea integradora responde a las expectativas del estudiante?, ¿La tarea integradora promueve la reflexión y el pensamiento crítico?, ¿La tarea integradora necesita ayuda de otros compañeros o del profesor para su comprensión y solución?, ¿Para abordar la tarea integradora se necesita ayuda externa?, ¿Cuál será la estrategia de evaluación?, ¿Cuáles instrumentos de evaluación son pertinentes emplear?, ¿Cuáles serán los criterios para valorar y medir el accionar del estudiante?, ¿Cuáles serán las técnicas para recabar la información? y ¿Desde cuál marco de referencia teórico se analizarán e interpretaran la información recabada?.

\section{REFLEXIONES FINALES}

1.- Las situaciones de aprendizaje reconocen al estudiante como sujeto activo capaz de lograr autonomía para aprender y del profesor como orientador de procesos que promuevan la formación de manera pertinente a las exigencias del contexto.

2.- Las situaciones de aprendizaje serán flexibles y favorecerán los saberes derivados de las competencias genéricas y específicas contempladas en el diseño curricular respectivo. Para ello debe considerar cómo, cuándo y qué aprende el estudiante.

3.- Las situaciones de aprendizaje son elementos constitutivos de la planificación que el profesor realiza para desarrollar el contenido de las unidades curriculares pertenecientes a un diseño curricular específico, para que los estudiantes discutan los contenidos y construyan saberes.

4.- Las situaciones de aprendizaje podría contemplar para su diseño los siguientes componentes aspectos técnicos - pedagógicos: (a) contexto; (b) ambiente de aprendizaje; (c) procesamiento de la información; y (d) tareas integradoras.

5.- Las situaciones de aprendizaje se caracterizan por generar acciones cognitivas, afectivas y emocionales en los estudiantes pertinentes para aprender en sintonía con la autoconsciencia, la cual es activada y mediada por la propia situación. En este sentido, el enfoque del aprendizaje estratégico y sus principios esenciales como lo son la metacognición y las estrategias de aprendizaje juegan un papel importante para la construcción autónoma del aprendizaje en un contexto educativo. 


\section{REFERENCIAS}

Ausubel, D., Novack, J., Hanesian, H. (1996). Psicología Educativa, un Punto de Vista Cognoscitivo. México: Trillas.

Batista, J. y Oliveira, E. (1976). Tecnología Educacional y Teorías de Instrucción. Buenos Aires: PAIDOS.

Chan, M. y Tiburcio, A. (2000). Guía para el Diseño de Materiales Educativos Orientados al Aprendizaje Autogestivo. México: INNOVA.

Conferencia Mundial sobre Educación Superior (2009). La Nueva Dinámica de la Educación Superior y de Investigación, para el Cambio Social y Desarrollo. [Documento en línea]. Disponible: http://www.unesco.org/es

Declaración Mundial sobre Educación Superior en el Siglo XXI (1998). [Documento en línea]. Disp.: http://www.oei.es

Feo, R. (2014). Diseño de Situaciones de Aprendizaje: Una Visión Desde y para la Educación A Distancia. Caracas: Mesa de trabajo Diseño de Situaciones de Aprendizaje para la Comisión Nacional Educación a Distancia UPEL.

Feo, R. y Guerra, C. (2014). Propuesta de un Modelo de Diseño Instruccional para la Elaboración e Implementación de Cursos a Distancia. Sapiems, 14, 1 22.

Flores, V. (2009). Planeación por Proyectos. [Blog en Línea]. Disponible: http://academiasefcnayavi.blogspot.com.

Gonzales, V., Gonzales, R., y López, A. (2011). Diseño de Situaciones de Aprendizaje que Potencien Competencias Profesionales en la Enseñanza Universitaria. Revista de Formación del Profesorado e Investigación Educativa, 24 (1), 121 - 134.

Herrera Clavero, F. (1991). Situaciones de aprendizaje-enseñanza España: Universidad de Granada, Instituto de Estudios Ceutíes.

Informe sobre Tendencias Sociales y Educativas en América Latina. Desafíos y Oportunidades: Metas Educativas 2021. (2010). [Documento en línea]. Disponible: http://www.siteal.iipe-oei.org

Marzano R. (1999). Dimensiones del Aprendizaje. México: ITESO.

Mendia, R. (1989). Cómo Definir una Situación de Aprendizaje. Eskola, 22, 1 - 12.

Leontiev, N. (1978). Actividad, Conciencia y Personalidad. California: PrenticeHall. (Traducción del ruso a inglés: Marie J. Hall).

Pérez, R. y López, F. (2000). Hacia Una Educación de Calidad, Gestión, Instrumentos y Evaluación. Madrid: Narcea.

Piraval, M., Morales. B., y Gutiérrez. M. (2013). Situaciones de Aprendizaje, Pautas Metodológicas Para el Desarrollo de Competencias en el Aula. Guatemala: dirección General de Currículo. 


\section{ANEXO \\ Situación de Aprendizaje Centrada en el Aprendizaje Estratégico}

\begin{tabular}{|c|c|}
\hline \multicolumn{2}{|c|}{$\begin{array}{l}\text { CURSO: Desarrollo de Procesos Cognoscitivos (DPC 0113) } \\
\text { MODALIDAD: Presencial - Quincenal } \\
\text { PERÍODO ACADÉMICO } 2015 \text { - II } \\
\text { NÚMERO DE ESTUDIANTES: } 17 \text { hembras - } 13 \text { varones } \\
\text { EDAD: } 18 \text { - } 50 \text { AÑOS } \\
\text { Competencia Específica: Valora al ser humano desde sus perspectivas } \\
\text { de acción, en el desarrollo de procesos de construcción, gestión y actuación de } \\
\text { saberes de la praxis educativa, en el marco de situaciones de estudio a fin de } \\
\text { generar la formación del pensamiento autónomo, crítico, reflexivo y ético. }\end{array}$} \\
\hline C & $\begin{array}{l}\text { pertenecen al tercer período académico sus edades } \\
\text { peros en } 30 \text { estudiantes inscritos en el curso } \\
\text { oscilan entre los } 18 \text { años y los } 5^{\circ} \text { años. Son activos y muy } \\
\text { vivos. La mayoría se conocen de otros cursos o } \\
\text { pertenecen a la misma especialidad. Existen tres } \\
\text { estudiantes sordos, doce estudiantes de educación física, } \\
\text { tres de Geografía e Historia, dos de Educación Musical, el } \\
\text { resto pertenecen a otras especialidades como Lengua } \\
\text { Castellana, Matemática, Física e Industrial. La primera } \\
\text { sección de presentación del curso y del plan de evaluación } \\
\text { surgieron preguntas que orientan al profesor a afirmar que } \\
\text { los estudiantes tienen curiosidad sobre los contenidos del } \\
\text { curso, lo que les motiva a estudiarlo. Los estudiantes } \\
\text { provienen del centro de Caracas y el de Guarenas - } \\
\text { Guatire, se trasladan en transporte urbano y tienen } \\
\text { celulares inteligentes. Su forma de vestir es una mezcla de } \\
\text { casual y deportiva. } \\
\text { - - Las situaciones de aprendizaje se darán bajo una } \\
\text { administración presencial - quincenal, lo que significa que } \\
\text { solo se tienen ocho semanas de encuentros pedagógicos } \\
\text { presenciales y el resto a distancia. Por las características } \\
\text { descritas se empleará el salón de clases denominado aula } \\
\text { 205 para los encuentros presenciales y para la interacción } \\
\text { a distancia se empleará un aula virtual alojada en el salón } \\
\text { virtual de la UPEL bajo la plataforma Moodle. Esta aula } \\
\text { virtual tendrá el mismo nombre del curso. } \\
\text { c- Se desconocen las estrategias de aprendizaje de } \\
\text { cada sujeto, pero el ambiente de aprendizaje y las } \\
\text { situaciones de aprendizaje las fomentaran en el estudiante } \\
\text { a través de la mediación del profesor. } \\
\text { del estudiante, indagando entre sus compañeros y en el } \\
\text { primer encuentro, bajo la técnica de la observación } \\
\text { participante. }\end{array}$ \\
\hline $\begin{array}{l}\text { AMBIENTE DE } \\
\text { APRENDIZAJE }\end{array}$ & $\begin{array}{l}\text {. - Por ser un curso bajo una modalidad presencial - } \\
\text { quincenal (Mixto) se debe prever de un espacio para los } \\
\text { encuentros pedagógicos presenciales que origina una } \\
\text { estrategia para consolidar los aprendizajes o dependiendo }\end{array}$ \\
\hline
\end{tabular}




\begin{tabular}{|c|c|}
\hline & $\begin{array}{l}\text { de los acontecimientos del curso para presentar los } \\
\text { contenidos y ejercitar. Igualmente, se necesita una } \\
\text { estrategia que se centre en mantener la expectativa del } \\
\text { estudiante o que estudie continuamente en provecho del } \\
\text { tiempo que no va físicamente a las clases (horas de labor } \\
\text { estudiantil). En este caso específico se diseñó un aula } \\
\text { virtual en el Salón Virtual UPEL bajo los principios del } \\
\text { diálogo didáctico mediado y el enfoque del aprendizaje } \\
\text { estratégico lo que generó un aula virtual que invita a la } \\
\text { interacción, al trabajo colaborativo y la autonomía escolar. } \\
\text { El link de acceso al aula virtual es: } \\
\text { http://salonvirtual.upel.edu.ve/course/view.php?id=749 } \\
\text { to - En referencia a los contenidos se asumen que } \\
\text { todos podrían fomentar aprendizaje estratégico, desde los } \\
\text { procesos básicos, los fundamentos teóricos, los seis } \\
\text { sombreros para pensar y las herramientas del } \\
\text { pensamiento. } \\
\text { materiales digitales en PDF y videos acompañados con } \\
\text { consignas del profesor. Se espera que esta combinación } \\
\text { pueda generar aprendizaje en los estudiantes o por lo } \\
\text { menos iniciarlos en él. En las clases presenciales se } \\
\text { abordará cada contenido desde una visión de asesoría, de } \\
\text { compartir experiencias para complementar lo aprendido en } \\
\text { el aula. Como medio didáctico se empelarán la pizarra, el } \\
\text { video Beam, pendones, libros. } \\
\text {. Las acciones formativas serán elementales, con } \\
\text { énfasis en la presencialidad en el salón de clases. El aula } \\
\text { virtual ofrecerá el espacio ideal para adjuntar las tareas o } \\
\text { productos finales para ser evaluados con ponderación. }\end{array}$ \\
\hline $\begin{array}{l}\text { PROCESAMIENTO } \\
\text { DE LA } \\
\text { INFORMACIÓN }\end{array}$ & $\begin{array}{l}\text { - Los procesos cognitivos a fomentar en el } \\
\text { estudiante serán el análisis, la síntesis, la comparación, la } \\
\text { clasificación y seguir instrucciones como proceso esencial } \\
\text { y capaz de apoyar el procesamiento de la información, } \\
\text { adicionalmente el estudiante tendrá consignas que le } \\
\text { invitaran a la reflexión sobre lo realizado para aprender los } \\
\text { contenidos del curso. Ese proceso de reflexión estará } \\
\text { presente en la mayoría de los instrumentos evaluativos, en } \\
\text { los foros del aula virtual, en las asesorías y conversaciones } \\
\text { cara a cara entre el profesor y el estudiante. }\end{array}$ \\
\hline $\begin{array}{l}\text { TAREA } \\
\text { INTEGRADORA }\end{array}$ & $\begin{array}{l}\text { va - Las tareas integradoras sintetizan lo aprendido y } \\
\text { va de mano con la estrategia de evaluación, es la arista } \\
\text { visible de este proceso. Las tareas integradoras estarán } \\
\text { acompañadas por foros que permitan socializar las } \\
\text { experiencias de aprendizaje del sujeto en vez de ocultarla, } \\
\text { lo que permitirá establecer juicios valorativos de mayor } \\
\text { aproximación a la realidad del estudiante y no en el } \\
\text { producto de la tarea. Se valora el desempeño del } \\
\text { estudiante. } \\
\text {. - Cada contenido tendrá una actividad afín a lo }\end{array}$ \\
\hline
\end{tabular}




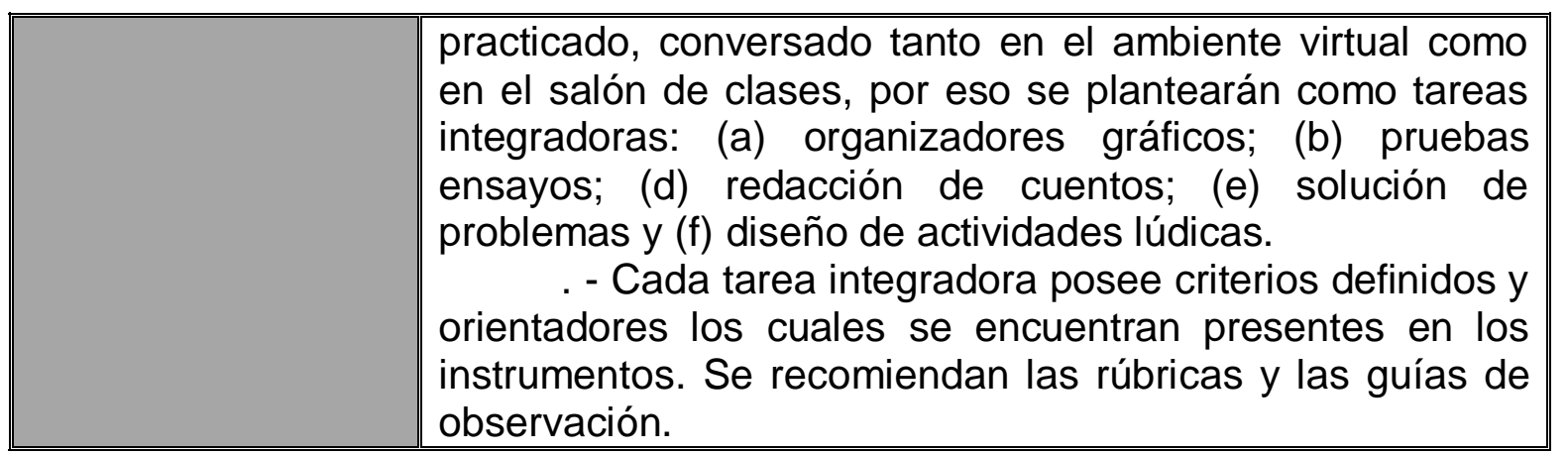

